\title{
Communicating with Patients with Disability: Perspectives of Practicing Physicians
}

\author{
Nicole Agaronnik, $B S^{7}$, Eric G. Campbell, $P h D^{2}$, Julie Ressalam, MPH, CHES ${ }^{2}$, and \\ Lisa l. lezzoni, MD, MSc ${ }^{1,3}$ \\ 'Mongan Institute Health Policy Center, Massachusetts General Hospital, Boston, MA, USA; ${ }^{2}$ Center for Bioethics and Humanities, University of \\ Colorado School of Medicine, Aurora, CO, USA; ${ }^{3}$ Department of Medicine, Harvard Medical School, Boston, MA, USA.
}

\begin{abstract}
BACKGROUND: Patient-centered care for people with disability requires effective communication and compliance with the Americans with Disabilities Act (ADA).

OBJECTIVE: To understand physicians' perspectives on communication experiences with people with disability.

DESIGN: Twenty semi-structured individual interviews. Interview recordings were transcribed verbatim for analysis.
\end{abstract}

SETTING: Massachusetts, October 2017-January 2018. PARTICIPANTS: Twenty physicians ranging from 8 to 51 years in practice in primary care or 4 other specialties. MEASUREMENTS: Commonly expressed themes around communication with people with disability.

RESULTS: Concerns coalesced around 4 broad categories: communication experiences with people who are deaf or hard of hearing, communication with people who are blind or have vision impairment, communication with people who have intellectual disability, and recommendations for improving communication. Although participants in this study reported various efforts to communicate effectively with patients with hearing or vision loss or intellectual disability, many gaps appear to remain, as well as instances where physicians' preferences run counter to patients' wishes and the ADA. Examples include physicians' preferences for remote, online sign language interpreters despite patients desiring in-person interpreters and suggesting that patients arrange for their own interpreters. Few educational materials are available in braille, and electronic medical records may not allow documents to be printed in large font for persons with low vision. Communicating with patients with intellectual disability raised particular concerns, with participants often preferring to interact with caregivers and minimal efforts to involve patients.

CONCLUSIONS: Effective communication is necessary for ensuring the quality of health care for people with disability, and it is legally required under the ADA. Our results suggest that important gaps may remain in ensuring effective communication, and some practicing physicians could benefit from formal training in effective methods for communicating with patients with disability.

Electronic supplementary material The online version of this article (https://doi.org/10.1007/s11606-019-04911-0) contains supplementary material, which is available to authorized users.

Received August 14, 2018

Revised November 13, 2018

Accepted January 24, 2019

Published online March 18, 2019
KEY WORDS: disability; communication; deaf; hard of hearing; blind; vision impairment; intellectual disability; Americans with Disabilities Act.

J Gen Intern Med 34(7):1139-45

DOI: $10.1007 /$ s11606-019-04911-0

(c) Society of General Internal Medicine 2019

\section{INTRODUCTION}

The 2011 World Report on Disability called for eliminating barriers to health care service delivery for persons with disability, including ensuring effective communication. ${ }^{1}$ Clinicians frequently cite communication concerns as a challenge to providing high-quality health care. ${ }^{1}$ A nationally representative survey, including $8.4 \%$ self-reporting disability, found that persons with disability noted problems communicating with clinicians significantly more often than persons without disability; concerns included failures of physicians to listen to patients' preferences or engage them sufficiently in decisionmaking. ${ }^{2}$ Within the diverse population of persons with disability, communication barriers are especially salient for individuals who are deaf or hard of hearing, are blind or have low vision, or have intellectual disability. Approximately 37.5 million US adults aged 18 and over report trouble hearing, ${ }^{3}$ approximately 25.5 million American adults aged 18 and over report some vision loss, ${ }^{4}$ and approximately 15.5 million Americans of all ages have an intellectual disability. ${ }^{5}$ All general internists can expect to see patients with one or more of these disabilities in their practice.

The Americans with Disabilities Act (ADA) requires that clinicians ensure effective communication with persons with disability, although it does not mandate specific communication modalities to achieve this goal. Instead, the ADA requires that patients' preferences be prioritized when choosing among communication options - such as American sign language (ASL) interpreters, communication access real-time translation (CART), or auxiliary aids for persons with hearing deficits, ${ }^{6}$ and qualified readers, braille materials, and enlarged fonts for persons with low vision, ${ }^{7}$ and various techniques for communicating with persons with intellectual disability. ${ }^{8}$ More specifically, ADA Title II entities (practices receiving funding from state or local governments) are legally required to give primary consideration to patients' preferences, while 
Title III entities (private practices serving the public) are encouraged to consult patients and emphasize their needs. ${ }^{9}$

Thus all practices, regardless of their ownership, must be prepared to accommodate diverse communication needs of their patients with disability. However, little is known about the extent to which practicing physicians have resources available to ensure effective communication. This study aimed to explore perceptions of practitioners about communicating with their patients with hearing, vision, or intellectual disability.

\section{METHODS}

The Massachusetts General Hospital (MGH)/Partners Healthcare Institutional Review Board (IRB) approved this study. The IRB viewed interviewees' willingness to participate after being told verbally about interview procedures, including audio-recording, as indicating informed consent.

\section{Interview Protocol}

This report comes from a larger study exploring physicians' experiences with and perceptions of serving patients with different disabilities. We designed an open-ended interview protocol (supplementary online appendix), drawing upon the literature and our previous studies. The protocol contained question modules addressing different disability-related topics, including communication. The first three interviews served as pilot interviews. Since we subsequently made only minor changes to the interview protocol, we included these first three interviews in the analysis.

\section{Participant Recruitment and Interview Procedures}

We aimed to recruit 25 physicians within disciplines where persons with disability frequently require some type of accommodation. These specialties included primary care (general internists and family practitioners), rheumatology, neurology, obstetrics/gynecology (OB/GYN, women in late pregnancy), and orthopedics. From a commercial source (SK\&A Healthcare Databases, Irvine, CA), we obtained contact information from randomly selected physicians in these fields practicing in Massachusetts $(n=520)$. We excluded fellows, residents, and other trainees. We also excluded physicians affiliated with Partners HealthCare (remaining $n=365$ ) due to concerns about response bias and administrative considerations. We randomly selected physicians for contact via email with telephone follow-up. Non-participation rates were difficult to assess because of missing and inaccurate contact information in the data source, and it is unclear whether certain recruitment emails were ignored or not seen by the intended recipient. Two physicians agreed to participate but subsequently were unreachable to schedule an interview. Recruitment concluded after 20 physicians due to data saturation (i.e., no new information emerged from additional interviews).
Audiotaped telephone interviews averaged $41 \mathrm{~min}$; no persons were present during the interview other than the participant and interviewer. No repeat interviews were conducted with any participant; we did not send transcripts to participants post-interview; and only minor notes were made postinterview (e.g., about interview logistics). We offered $\$ 100$ to each interviewee for participation, but 4 refused viewing their participation as a donation. N.A. compared audio files to transcriptions and made minor corrections.

\section{Research Team and Reflexivity}

Here, we report items consistent with the COnsolidated criteria for REporting Qualitative Research checklist. L.I.I. conducted all interviews. The research team included L.I.I. (MD, MSc, professor, health services researcher) and N.A. (research assistant) based in Boston and E.G.C. (PhD, professor, survey scientist) and J.R. (MPH, project manager) at the University of Colorado School of Medicine in Denver. The team included three individuals who identify as female and one as male. All researchers had experience and/or training in qualitative research methods. The interviewer (L.I.I.) has extensive experience conducting in-depth, qualitative research interviews on topics relating to disability. She has been a member of the local medical and research community for more than 30 years and is a nationally known figure in the field of disability research; therefore, not surprisingly, four of the randomly selected interviewees were acquainted with L.I.I. However, she has not had contact or correspondence with these individuals in several years. All four of these interviewees reported experiences and perceptions that reflected negatively on disability accommodations within their institutions; therefore, L.I.I. did not feel they exhibited a positive response bias because of their acquaintance with her. None of these individuals had prior knowledge of this study, but they were informed that the interview goals were to learn about their experiences with and perceptions of caring for persons with disability. The research team has no personal biases that might have influenced the outcomes of this study.

\section{Analysis}

As our analytic approach, we used conventional content analysis, a qualitative analysis method that draws explicitly from the data without overinterpretation of results. ${ }^{10-12}$ For this analysis, we focused on responses to the interview protocol module relating to communication. After reviewing the transcripts multiple times, coding categories were generated to facilitate analysis. N.A. initially coded the data using word processing software; for these descriptive studies, the researchers have found that coding in this fashion is more efficient than involving qualitative software (NVivo, Atlas.ti, etc.). The two Boston researchers (L.I.I. and N.A.) met frequently to reach initial consensus on the coding and findings. Independently, the two Denver researchers (E.G.C. and J.R.) reviewed the transcripts and identified the major findings. The 
two teams then met by teleconference call and reviewed the results identified independently by each group, reaching consensus about identified themes and patterns. Below, we sometimes indicate numbers of interviewees reporting specific experiences to avoid vague language (e.g., "some," "several," "many").

\section{RESULTS}

Table 1 presents demographic information about the 20 participants from different clinical practices. The mean (S.D.) time in practice was 27.4 (12.5) years. Results below contain quotes that exemplify responses, while Appendix Tables 1 and 2 in the Electronic Supplementary Material (ESM) contain additional quotations not mentioned in the text.

\section{Communicating with Patients Who Are Deaf or Hard of Hearing}

Themes concerning communication with patients who are deaf or hard of hearing were as follows: physicians frequently utilized communication modalities other than ASL interpreters or auxiliary aids, communication preferences varied across physicians, and physicians' preferences did not always align with patients' preferences. Thirteen physicians had access to ASL interpreters, and 10 had TTY/TDD (teletypewriters) in their practice. Physicians reported using other approaches to communicate with patients who are deaf or hard of hearing without involving ASL interpreters or auxiliary aids, including writing notes, lip reading, changing pitch, and talking at a slower speed. An internist who reported adjusting her pitch

Table 1 Characteristics of Interviewees, $N=\mathbf{2 0}$

\begin{tabular}{ll}
\hline \hline Characteristic & \\
\hline Age, mean (S.D.) years & 53.5 \\
Age range (years) & $(11.7)$ \\
Gender, $n$ & $38-76$ \\
Male & \\
Female & 10 \\
Race, $n$ & 10 \\
White & 18 \\
Non-white & 2 \\
Hispanic ethnicity, $n$ & 1 \\
Specialty, $n$ & \\
Internal medicine & 7 \\
Family practice & 1 \\
Rheumatology & 2 \\
Neurology & 6 \\
Obstetrics/gynecology & 2 \\
$\quad$ Orthopedics & 2 \\
Time in practice, mean (S.D.) years & 27.4 \\
& $(12.5)$ \\
Type of practice, $n$ & 16 \\
Hospital-based & 4 \\
Private, not hospital-based & \\
Communication methods available in practice, $n$ & 13 \\
Has access to American sign language (ASL) interpreters & 10 \\
Has access to teletypewriter (TTY/TDD) & 10 \\
Has at least some informational material available in & 5 \\
large font & \\
Has at least some informational material available in \\
braille & 1 \\
\hline
\end{tabular}

conceded "I don't know if it helps. It makes me feel like I'm doing something..." Her clinical practice had not previously considered getting auxiliary aids. A rheumatologist, in practice for more than 30 years, recommended "shouting into their good ear." Appendix Table 1 (ESM) shows other exemplary quotations.

Physicians frequently attempted written communication. An internist said "Writing it out is helpful because the patients kind of can follow along." However, some physicians described the inefficiency of written communication: "It's slow. I think we probably are not providing as much detail as we ordinarily would be."

Communication preferences varied by physician. One rheumatologist, for whom ASL interpreters were not her first choice, relied upon a video interpreter system. Another physician preferred in-person ASL interpreters:

...there's a lot of unspoken communication that occurs... there's unspoken empathy...patients understand the tonality and the intent of what I say better when there's a live, other person in the room ... We also have to use the phone [TTY/TDD] for some of these, and that's brutal ...you really lose all of the sort of non-verbal cues...

Physician and patient preferences sometimes differed. A neurologist, whose patients preferred having a live ASL interpreter, disliked interpreters because "They're late. They're hard to find..." An internist concurred that it was logistically challenging to arrange for ASL interpreters, so she frequently relied upon a video interpretation instead: "if you have the computer, you can do it that day, but again, the patient doesn't feel well represented... The patient really prefers the real person."

\section{Communication with Patients Who Are Blind or Have Vision Impairment}

Themes concerning communication with patients who are blind or have vision impairment were as follows: physicians had a paucity of accessible informational material, and communication challenges were amplified with patients who were deaf-blind or had multiple disabilities.

Five participants reported having accessible informational materials (e.g., large font) for patients with vision impairment. Only 1 physician reported having any materials available in braille (see Appendix Table 2 in ESM). A neurologist indicated that the hospital had braille versions for "generic consents to be treated at the hospital. We don't have Braille available for disease-specific information."

Five reported having informational materials in large font, especially online items allowing fonts to be adjusted before printing. However, an internist described frustration with adjusting font in the electronic medical record (EMR): "That has been a battle. So, I will write it out with a big sharpie." 
Physicians who relied upon commercially prepared materials, such as prepared pamphlets, explained that large font was not available. Physicians offered other reasons for not having informational material available in accessible format, but as an internist noted, "I don't really have any solutions for it because we never needed them." Two participants suggested that materials could be found online.

One participant described unique challenges with patients who are deaf-blind, as reported by a neurologist: "If patients came in hearing impaired but now they have a new deficit that...might make them now newly visually impaired...So using the sign language interpreter when they can't see...It's very difficult."

\section{Communication with Patients Who Have Intellectual Disability}

Themes concerning communication with patients who have intellectual disability were as follows: physicians had variable, unstandardized approaches for communication with patients, physicians frequently did not directly engage the patient due to the physician's estimation of the patient's decision-making ability, physicians preferred to communicate with caregivers despite concerns over patient consent, and some physicians used visual aids to assist in communication.

Eighteen participants described communication with patients who have intellectual disability. Only 4 participants reported that they initially try to communicate directly with the patient in a way that the patient would be able to understand. An internist described the communication strategy as "pretty individualized," while another internist stated "I actually directly talk to them." However, some physicians were not sure about the appropriate approach: "The first sitting, you're caught off guard... But once you know the patient... you curb the way you speak..." Another physician agreed: "You try to wipe away the jargon ... the patients probably is not understanding very much and too proud to interrupt."

Most of the time, physicians did not engage the patient with intellectual disability directly. Physicians almost always included family members and caretakers in the dialogue, often without focusing on the patient's experience. For example, an internist asserted "I tend to rely on those other caregivers to help with the explanations, but also with some of the decisionmaking if that's their role... I think where I'm not totally comfortable is if the patient has full decision-making power..." A rheumatologist concurred that patients with intellectual disability "are not able to make decisions for themselves, and that they usually still need a parent to be there with them." The rheumatologist described a patient whom she has not previously asked about decision-making because he always brings a parent to the clinic, conceding that "I need to be more aware of that."

Physicians emphasized that it is helpful to have caregivers assist with communication during appointments. An internist suggested "If there's any concern, or if there is any social thing that I need to confirm with the patient, then I got to the PCA." Another internist described a similar benefit:

...It's very much like trying to get information out of a little child who doesn't have very good verbal skills. People who are around that little kid every day learn what it is they're trying to say, even though an outsider won't be able to follow.

Though most physicians preferred to have a caregiver available to assist in communication, it was not always clear whether the patient consented. For example, an internist described a situation where the patient's preference was unclear:

I don't think we actually ask because the medical assistant puts the patient in the room. ... when they're already in the room, I assume that the discussion had happened...I should definitely pay more attention to it...

Having a caregiver present in the room without the patient's consent could have implications: "There is not a good way to screen for the abuse ... asking the caretaker does not really help.... It is very hard to get the right story... it was a little bit an uncomfortable discussion, especially with the caretaker..."

Seven participants described using visual aids, such as drawings and models, to communicate with patients with intellectual disability. However, one physician described models as "helpful for everybody," and that "there's more a spectrum rather than saying this group I don't need to explain anything to and this other group who has disabilities I need to explain a lot to."

\section{Communication Recommendations Proposed by Physicians}

Recommendations for improving communication with people with disability included more ASL interpreters and accessible informational material. One participant recommended placing responsibility for communication on the patient, such as arranging their own sign language interpreter to remove this responsibility from the practice:

...If I was in a country where I didn't speak the language, I would make an effort to have someone with me that could translate ... everybody has to bear some responsibility for the communication piece, and that some of that should arguably rely on the patients' efforts...

\section{DISCUSSION}

Effective communication is essential for achieving patientcentered care, and it is also required under the ADA. However, access barriers and assumptions about communication 
preferences create risks including inaccurate and incomplete medical information, medical errors (e.g., incorrect medication dosages), and misdiagnoses. ${ }^{13}$ Although participants in this study reported various efforts to communicate effectively with patients with hearing or vision loss or intellectual disability, many gaps appear to remain, as well as instances where physicians' preferences run counter to patients' wishes and the ADA.

In a previous study exploring the health care experiences of patients who are deaf or hard of hearing, patients believed that physicians were not aware of frequent communication barriers. ${ }^{13}$ Concurrent with findings in this study, physicians may also assume that communication via note writing and lip reading may be sufficient, even though this does not align with patient preferences or communication ability. ${ }^{14-16}$ Only $30-40 \%$ of spoken English can be understood through lip reading, ${ }^{16,17}$ suggesting that this method is unreliable especially for critical medical information. Writing may also be ineffective for some patients because of reading ability: the median reading proficiency of patients who are deaf or hard of hearing who have graduated from high school in the USA is between a fourth and fifth grade level, ${ }^{18}$ and their English vocabulary is similar to that of people for whom English is not their first language. ${ }^{19}$ Patients who are deaf or hard of hearing often prefer professional ASL interpreters or health care practitioners who are proficient in ASL. ${ }^{20,21}$

Section 504 of the Rehabilitation Act of 1973 and the ADA both require effective communication in a clinical setting unless accommodating a patient would result in an "undue hardship" for the clinical practice. However, the communication method is flexible: physicians are not required to have all communication mediums available in their practice, but they should be readily available if a patient requests a specific accommodation. ${ }^{22}$ However, not all physicians in this study reported access to frequently requested accommodations such as ASL interpreters or TTY/TTD. Furthermore, the responsibility for funding auxiliary aids cannot be placed onto the patient. Though none of the physicians in this study suggested that the cost of hiring ASL interpreters was a barrier in their clinical practice, this may be a concern for other physicians. The cost of hiring an ASL interpreter may sometimes exceed reimbursement, creating a financial disincentive for physicians to use ASL interpreters in their practice. ${ }^{23}$ It may be helpful to view the cost as an investment ${ }^{24}$ : it is highly likely that physicians will encounter patients with hearing impairment at some point in their practice. Preparedness can prevent law suits, as courts have previously held physicians accountable for failing to provide accommodations. ${ }^{25,26}$

Only 5 physicians identified having accessible reading material in large font, while only 1 physician had any informational material available in braille. The paucity of accessible reading material for patients who are blind or have low vision is antithetical to the goal of effective communication and in violation of the ADA as well as the Affordable Care Act (ACA). The ADA mandates effective communication in any form that is most appropriate for the patient, ${ }^{27}$ while Section 1557 of the ACA makes it a federal requirement that a clinical practice has alternative formats and auxiliary aids in order to be accessible for all patients. ${ }^{28}$ Auxiliary aids may include as follows: braille, taped text, and qualified readers. Brailtalk is an inexpensive device that has a raised alphabet and characters, and the physician can guide the patient through touching the appropriate letters or characters. Help cards with premade braille messages may also be used to communicate simple information. ${ }^{27}$ Physicians should ensure that all information provided via the Internet should have a screen reader functionality in compliance with Section 508 of the Rehabilitation Act of $1973 .{ }^{29}$ Guides for effective communication with patients who have vision impairment are also available. ${ }^{30}$

Communicating with patients who have intellectual disability was especially challenging, and several participants struggled with determining when it was necessary to have a caretaker involved in communication. Patients with intellectual disability report feeling excluded from dialogue about their care $^{31,32}$ and that physicians de-emphasize the decisionmaking ability of the patient. ${ }^{33,}{ }^{34}$ A survey of 440 internists suggested that they experience discomfort in treating patients with intellectual disability, and they often doubt the ability of such patients to make health care decisions. Physicians who had more experience with such patients generally communicated more directly with the patient. ${ }^{35}$ The United Nations Convention on the Rights of Persons with Disabilities asserted that everyone has the right to decision-making regardless of cognitive ability. Caretakers should not be making decisions in lieu of patients, but rather be available as a resource to support the patient's preferences. ${ }^{36}$

The ADA requires that effective communication is ensured for all patients including those with intellectual disability, and there are several tips, consistent with recommendations provided by respondents, that physicians can rely upon to improve their communication with such patients. ${ }^{37,38}$ Visual aids were one such recommendation that may be useful for explaining medical procedures. ${ }^{39}$

This study has several limitations. Though we reached data saturation, only 20 physicians were interviewed, limiting the generalizability of our findings. Given that we excluded Partners HealthCare, our results do not generalize to all physicians practicing in Massachusetts or the USA. Results are also not generalizable to each subspecialty and practice type. The mean age of participants was in the early 50s; therefore, these results may not generalize to a younger generation of physicians who were trained after passage of the ADA and may know more about accommodating people with disability and their legal responsibilities under the ADA. It is important to note, however, that few medical school curriculums contain training about disability civil rights and required accommodations. Another potential limitation is that four randomly recruited participants were acquainted with the interviewer, although without any contact in at least several years. All four interviewees shared concerns about disability accommodations in 
their practice environments, reducing concerns about positive response biases. These particular individuals may have felt comfortable sharing these concerns, given their prior acquaintance with the interviewer.

We limited the number of questions that were asked so that we could obtain a deeper contextual understanding of communication experiences with patients who have various disability experiences, focusing on patients who are deaf or hard of hearing, are blind or have visual impairment, or have intellectual disability. However, this does not represent the full diversity of disability experiences that may pose unique challenges for communication. Though one physician described a communication experience with a patient who was deaf-blind, more investigation is required into communication needs for patients with multiple disabilities. Furthermore, we were not expecting so few participants to report having accessible reading material or other accommodations for patients with vision impairment. This invites a more in-depth exploration of physician experiences with accommodating patients who have vision impairment in their practice, as well as more concrete inquiries into the availability of specific auxiliary aids.

Barriers to effective communication appear to be a universal problem across disability experiences. Though federal law does not legally mandate specific communication methods, physicians are required to accommodate patients based on patients' personal preference, creating an incentive to be prepared for addressing a diversity of needs. Furthermore, it is critical that physicians engage patients in decision-making to ensure autonomy over their health care experience. Our results suggest that more physician education is required about effectively communicating with patients with disability.

\section{Acknowledgements:}

We thank Colin Ponzani for his assistance with recruitment of interview participants.

Funding/Support: This work was funded partially by Deliberative Interim Support Funding from the Executive Committee on Research, Massachusetts General Hospital, and partially by the Eunice Kennedy Shriver National Institute of Child Health and Human Development R01 HD091211-01A1.

Corresponding Author: Lisa I. Iezzoni, MD, MSc; Mongan Institute Health Policy Center, Massachusetts General Hospital, Boston, MA, USA (e-mail: liezzoni@mgh.harvard.edu).

Compliance with Ethical Standards:

The Massachusetts General Hospital (MGH)/Partners Healthcare Institutional Review Board (IRB) approved this study.

Conflict of Interest: The authors declare that they do not have a conflict of interest.

Publisher's Note: Springer Nature remains neutral with regard to jurisdictional claims in published maps and institutional affiliations.

\section{REFERENCES}

1. The World Report on Disability. Geneva: World Health Organization and The World Bank; 2011.
2. Smith DL. Disparities in patient-physician communication for persons with a disability from the 2006 Medical Expenditure Panel Survey (MEPS). Disabil Health J. 2009;2(4):206.

3. National Institute on Deafness and Other Communication Disorders. Quick Statistics about Hearing. https://www.nidcd.nih.gov/health/statistics/quick-statistics-hearing. Accessed January 10, 2019.

4. American Foundation for the Blind. Facts and Figures on Adults with Vision Loss. http://www.afb.org/info/blindness-statistics/adults/factsand-figures/235\#demographics. Published 2018. Accessed January 10, 2019.

5. Cornell University Yang Tan Institute on Employment and Disability. Disability Statistics. http://www.disabilitystatistics.org/reports/acs. cfm?statistic=1. Accessed January 10, 2019

6. Civil Rights Division, Department of Justice. ADA business brief: communicating with people who are deaf or hard of hearing in hospital settings. https://www.ada.gov/hospcombr.htm. Published 2003. Accessed January 10, 2019.

7. ADA National Network. What kinds of auxiliary aids and services are required by the $\mathrm{ADA}$ to ensure effective communication with individuals with hearing or vision impairments? https://adata.org/faq/what-kindsauxiliary-aids-and-services-are-required-ada-ensure-effective-communication. Accessed January 10, 2019.

8. Informed consent in adults with developmental disability. https://www. cfpc.ca/uploadedFiles/Directories/Committees_List/Informed Consent in Adults with DD.pdf. Published 2009. Accessed January 10, 2019.

9. Civil Rights Division, Department of Justice. ADA requirements effective communication. https://www.ada.gov/effective-comm.htm. Published 2014. Accessed January 10, 2019.

10. Sandelowski M. Focus on research methods: Whatever happened to qualitative description? Res Nurs Health. 2000;23:334-340.

11. Sandelowski M. What's in a name? Qualitative description revisited. Res Nurs Health. 2010;33(1):77-84. https://doi.org/10.1002/nur.20362.

12. Hsieh H-F, Shannon SE. Three approaches to qualitative content analysis. Qual Health Res. 2005;15(9):1277-1288. https://doi.org/10. $1177 / 1049732305276687$.

13. Iezzoni LI, O'Day BL, Killeen M, Harker H. Communicating about health care: observations from persons who are deaf or hard of hearing. Ann Intern Med. 2004; 140(5):356-362+I68.

14. Barnett S. Clinical and cultural issues in caring for deaf people. Fam Med. 1999;31(1):17-22.

15. Barnett S. Cross-cultural communication with patients who use American Sign Language. Fam Med. 2002;34(5):376-382.

16. Ebert DA, Heckerling PS. Communication with deaf patients. Knowledge, beliefs, and practices of physicians. JAMA. 1995;273(3):227-229.

17. Steinberg A. Issues in providing mental health services to hearingimpaired persons. Hosp Community Psychiatry. 1991;42(4):380-389.

18. Holt JA. Stanford Achievement Test-8th Edition: reading comprehension subgroup results. Am Ann Deaf. 1993;138(2):172-175.

19. McEwen E, Anton-Culver H. The medical communication of deaf patients. J Fam Pract. 1988;26(3):289-291.

20. Steinberg AG, Barnett S, Meador HE, Wiggins EA, Zazove P. Health care system accessibility: experiences and perceptions of deaf people. J Gen Intern Med. 2006;2 1(3):260-266.

21. Mackinney TG, Walters D, Bird GL, Nattinger AB. Improvements in preventive care and communication for deaf patients: results of a novel primary health care program. J Gen Intern Med. 1995;10(3): 133-137.

22. National Association of the Deaf. Questions and answers for health care providers. https://www.nad.org/resources/health-care-and-mentalhealth-services/health-care-providers/questions-and-answers-forhealth-care-providers/. Accessed January 10, 2019.

23. Iezzoni LI. Public health goals for persons with disabilities: looking ahead to 2020. Disabil Health J. 2009;2(3):111-115.

24. Kirschner KL, Breslin M Lou, Iezzoni LI. Structural impairments that limit access to health care for patients with disabilities. JAMA. 2007;297(10):1121-1125. https://doi.org/10.1001/jama.297.10.1121.

25. Harty-Golder B. Deaf services. The ADA and risk management. J Fla Med Assoc. 1996;83(8):573-576.

26. Requirements of ADA create challenges for providers. Hosp Law Newsl. 1995; 12(8): 1-4.

27. Marshall S, Joffee E. ADA Checklist: Health Care Facilities and Service Providers. American Foundation for the Blind. http://www.afb.org/info/ programs-and-services/public-policy-center/civil-rights/advocacy-resources/ada-checklist-health-care-facilities-and-service-providers / 12345. Accessed January 10, 2019.

28. Department of Health and Human Services. Section 1557: Ensuring effective communication with and accessibility for individuals with 
disabilities; 2010. https://www.hhs.gov/sites/default/files/1557-fs-disability-discrimination-508.pdf.Accessed January 10, 2019.

29. Department of Health and Human Services. Accessibility training: Introduction to accessibility and Section 508. https://www.hhs.gov/ sites/default/files/Intro to Accessibility and 508.pdf. Accessed January 10, 2019.

30. Schneider K. Caring better for patients who are blind or visually impaired. Am Fam Physician. 2013;88(11):774.

31. Ziviani J, Lennox N, Allison H, Lyons M, Del Mar C. Meeting in the middle: improving communication in primary health care consultations with people with an intellectual disability. J Intellect Dev Disabil. 2004;29(3):211-225.

32. Brown AA, Gill CJ. New voices in women's health: perceptions of women with intellectual and developmental disabilities. Intellect Dev Disabil. 2009;47(5):337-347.

33. Carlson D, Smith C, Wilker N. Devaluing people with disabilities: medical procedures that violate civil rights. National Disability Rights Network; 2012.

34. Huneke NTM, Gupta R, Halder N, Chaudhry N. Difficult decisions: are intellectually disabled patients given enough information to consent to medical treatment? J Intellect Disabil. 2012;16(4):265-274.
35. Werner S, Yalon-Chamovitz S, Tenne Rinde M, Heymann AD. Principles of effective communication with patients who have intellectual disability among primary care physicians. Patient Educ Couns. 2017;100(7):1314-1321.

36. United Nations. Convention on the rights of persons with disabilities. Treaty Ser. 2006;2515:3.

37. Riddle I, Romelczyk S, Sparling E. Effective communication for health care providers: a guide to caring for people with disabilities. Newark: Center for Disabilities Studies, University of Delaware; 2011. http:// www.cds.udel.edu/wp-content/uploads/2017/02/effective-communication.pdf. Accessed January 10, 2019.

38. McMillin T. Tips for facilitating effective communication between caregivers and individuals with IDD. https://www.relias.com/blog/tips-foreffective-communication-between-caregivers-and-individuals-with-idd. Published 2016. Accessed January 10, 2019.

39. Vanderbilt Kennedy Center for Excellence in Developmental Disabilities. Health care for adults with intellectual and developmental disabilities. https://vkc.mc.vanderbilt.edu/etoolkit/general-issues/communicatingeffectively/. Accessed January 10, 2019. 